\title{
The spectral energy distribution of CO lines in M 82
}

\author{
A. Weiß ${ }^{1}$, F. Walter ${ }^{2}$, and N. Z. Scoville ${ }^{3}$ \\ 1 IRAM, Avenida Divina Pastora 7, 18012 Granada, Spain \\ e-mail: aweiss@iram.es \\ 2 MPIA, Königstuhl 17, 69117 Heidelberg, Germany \\ e-mail: walter@mpia.mpg.de \\ ${ }^{3}$ Caltech, Pasadena, CA, USA \\ e-mail: nzs@astro.caltech.edu
}

Received 11 January 2005 / Accepted 7 April 2005

\begin{abstract}
We present an analysis of the excitation conditions of the molecular gas in the streamers and the outflow of M 82 based on observations obtained at the IRAM $30 \mathrm{~m}$ telescope. Our analysis of $J=1 \rightarrow 0$ and $J=2 \rightarrow 1$ transitions of CO and ${ }^{13} \mathrm{CO}$ and the $\mathrm{CO}(J=3 \rightarrow 2)$ line in 13 regions outside the central starburst disk shows that the gas density within the streamer/outflow system is about an order of magnitude lower $\left(\log \left(n_{\mathrm{H}_{2}}\right) \approx 3.0 \mathrm{~cm}^{-3}\right)$ than in the central molecular disk. We have used an LVG model and data from the literature to constrain the flux density in each CO transition (the "CO line SED") arising from the streamer/outflow system and the central starburst disk itself. Globally, we find that the CO flux density up to the $J=3 \rightarrow 2$ line is dominated by the diffuse outer regions while lines above the $J=5 \rightarrow 4$ transition are almost exclusively emitted by the central starburst disk. We compare the CO line SED of M 82 to CO observations of galaxies at high redshift and suggest that small high- $J$ /low- $J$ CO flux density ratios (observed in some of these sources) are not necessarily caused by a different excitation of the central molecular gas concentration, but may result from an additional, more extended and diffuse gas reservoir around these systems, reminiscent of the situation in M 82.
\end{abstract}

Key words. ISM: molecules - galaxies: halos - galaxies: high-redshift - galaxies: individual: M 82 - galaxies: ISM galaxies: starburst

\section{Introduction}

Studying the physical properties of the molecular gas in galaxies is of fundamental importance for understanding the processes leading to star formation at low and high redshifts. Due to its proximity and its strong emission in molecular lines, the nearby starburst galaxy M 82 is one of the best studied starburst environments. The central concentration of molecular gas, which feeds the strong star formation activity, has been the subject of many studies addressing both the excitation conditions and the distribution of the molecular gas (e.g., Young \& Scoville 1984; Wild et al. 1992; Weiß et al. 1999; Mao et al. 2000; Weiß et al. 2001; Ward et al. 2003). Some of these studies already have provided evidence for extended CO emission around M 82's center (e.g. Stark \& Carlson 1984; Seaquist \& Clark 2001; Taylor et al. 2001). A high-resolution, wide-field CO study revealed that large amounts of molecular gas are present in molecular streamers and the outflow around M 82's central disk (Walter et al. 2002).

Recent studies of CO line and dust continuum emission in distant quasar host galaxies $(z>2)$ have used M 82's central starburst disk as a nearby template (e.g. Yun et al. 2000; Cox et al. 2002; Carilli et al. 2002a,b; Weiß et al. 2003). At cosmological distances, however, only integrated values can be derived as usually no information on the spatial distribution of the molecular gas exists. Therefore $\mathrm{CO}$ line ratios can only provide information on the galaxies average, global excitation conditions. Consequently a comparison of such line ratios to values determined only for the center of M 82 are incomplete and biased as they neglect the contribution of a possible extended molecular gas component which could potentially have significantly different excitation conditions. This is the main motivation for the present study in which we present an analysis of the excitation conditions of the extended molecular gas surrounding M 82's central starburst disk.

\section{Observations and data reduction}

Using the IRAM $30 \mathrm{~m}$ telescope we observed the $\operatorname{CO}(J=$ $1 \rightarrow 0), \mathrm{CO}(J=2 \rightarrow 1),{ }^{13} \mathrm{CO}(J=1 \rightarrow 0)$ and ${ }^{13} \mathrm{CO}(J=2 \rightarrow 1)$ transitions towards 13 positions covering the molecular outflow and the molecular streamers surrounding M 82's starburst toroid (see Fig. 1). Spectra were obtained using the wobbler switch technique with a wobbler throw of $240^{\prime \prime}$ and a wobbler frequency of $0.5 \mathrm{~Hz}$. The receiver alignment was determined using pointing scans on Saturn and was found to be better than $2^{\prime \prime}$. Pointing was checked every $2 \mathrm{~h}$ on the nearby 


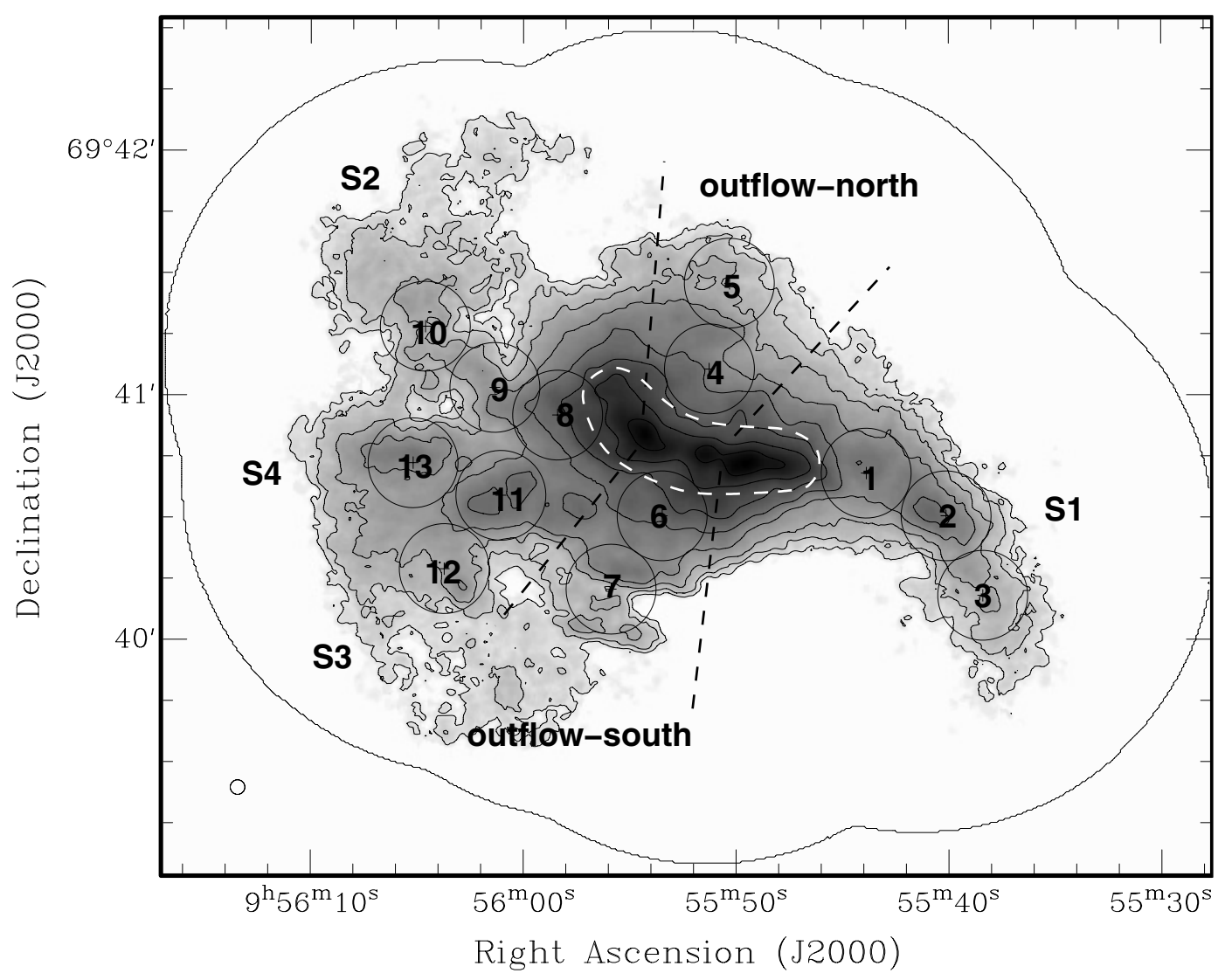

Fig. 1. Logarithmic representation of the integrated $\operatorname{CO}(J=1 \rightarrow 0)$ map of the zero-spacing-corrected OVRO mosaic (Walter et al. 2002). Circles indicate the observed positions for which we analyzed the CO line brightness temperature ratios. The size of the circles corresponds to $22^{\prime \prime}$, i.e. the spatial resolution of the $30 \mathrm{~m}$ telescope at $3 \mathrm{~mm}$. The white dashed contour shows our division between the central molecular disk and the streamer/outflow system.

quasars $0954+658$ and $0836+710$. We estimate the pointing accuracy to be better than $3^{\prime \prime}$. Calibration was checked once per observing run on $\mathrm{W} 3 \mathrm{OH}$ and the south-western molecular lobe in M 82.

System temperatures were on average $T_{\text {sys }} \approx 180 \mathrm{~K}$ and $330 \mathrm{~K}$ at 115 and $230 \mathrm{GHz}$ respectively. The data were recorded using the Vespa auto correlator with $512 \mathrm{MHz}$ bandwidth and $1.25 \mathrm{MHz}$ resolution at $3 \mathrm{~mm}$ and two $1 \mathrm{MHz}$ filter banks (512 channels each) at $1 \mathrm{~mm}$. The velocity coverage at $115 \mathrm{GHz}$ is $1330 \mathrm{~km} \mathrm{~s}^{-1}$ with $3.4 \mathrm{~km} \mathrm{~s}^{-1}$ resolution. The corresponding values at $230 \mathrm{GHz}$ are $660 \mathrm{~km} \mathrm{~s}^{-1}$ and $1.3 \mathrm{~km} \mathrm{~s}^{-1}$. The telescope's resolution at 230 and $115 \mathrm{GHz}$ is $11^{\prime \prime}$ and $22^{\prime \prime}$ respectively. To enable comparison between the brightness temperatures at 230 and $115 \mathrm{GHz}$ we observed a cross consisting of five pointings at each position (separation $\left.6^{\prime \prime}\right)$. Depending on the CO line strength the integration time at each central pointing was between 12 and $54 \mathrm{~min}$. All other pointings within the cross were observed for 6 or $12 \mathrm{~min}$.

For data processing we used the CLASS software package. Only linear baseline were removed from each spectrum. For the $\operatorname{CO}(J=1 \rightarrow 0)$ and the ${ }^{13} \operatorname{CO}(J=1 \rightarrow 0)$ lines only spectra from the central pointing were coadded. For the reduction of the $\operatorname{CO}(J=2 \rightarrow 1)$ and the ${ }^{13} \mathrm{CO}(J=2 \rightarrow 1)$ data, spectra at each pointing in the cross were coadded. From the resulting 5 spectra we generated the final spectrum at $22^{\prime \prime}$ resolution by a weighted addition with a weight of 1 for the central and 0.71 for the other four spectra (Johannsson et al. 1994). The coadded spectra were converted to the main beam brightness temperature scale $\left(T_{\mathrm{mb}}=F_{\mathrm{eff}} / B_{\mathrm{eff}} T_{\mathrm{A}}^{*}\right)$ using $F_{\text {eff }}=0.95, F_{\text {eff }}=0.91$ and $B_{\text {eff }}=0.71, B_{\text {eff }}=0.49$ at 115 and $230 \mathrm{GHz}$ respectively (IRAM newsletter 55, 2003). Finally, $\operatorname{CO}(J=1 \rightarrow 0)$ and $\operatorname{CO}(J=2 \rightarrow 1)$ spectra were smoothed to $3 \mathrm{~km} \mathrm{~s}^{-1}$ resolution. The final resolution of the ${ }^{13} \mathrm{CO}(J=1 \rightarrow 0)$ and ${ }^{13} \mathrm{CO}(J=2 \rightarrow 1)$ spectra is $10 \mathrm{~km} \mathrm{~s}^{-1}$. In addition to theses observations we used the $\mathrm{CO}(J=3 \rightarrow 2)$ data from Dumke et al. (2001). To allow a comparison to our data we smoothed the $\mathrm{CO}(J=3 \rightarrow 2)$ data cube to $22^{\prime \prime}$ resolution before extracting spectra at each position covered by our observations. Individual spectra at $22^{\prime \prime}$ resolution are shown in Fig. 2. Table 1 summarizes the line parameters at each position.

\section{Results}

\subsection{Line brightness temperature ratios}

The $\mathrm{CO}(J=1 \rightarrow 0), \mathrm{CO}(J=2 \rightarrow 1)$ and ${ }^{13} \mathrm{CO}(J=1 \rightarrow 0)$ lines are detected towards all positions. At position 13 (streamer $\mathrm{S} 4$ ) the ${ }^{13} \mathrm{CO}(J=2 \rightarrow 1)$ line is not detected $(3 \sigma$ upper limit: $\left.T_{{ }^{13} \mathrm{CO}(J=2 \rightarrow 1)}<20 \mathrm{mK}\right)$. The $\mathrm{CO}(J=3 \rightarrow 2)$ line is detected at all positions except positions 13 which is outside the field observed by Dumke etal. (2001). The line profiles of all 

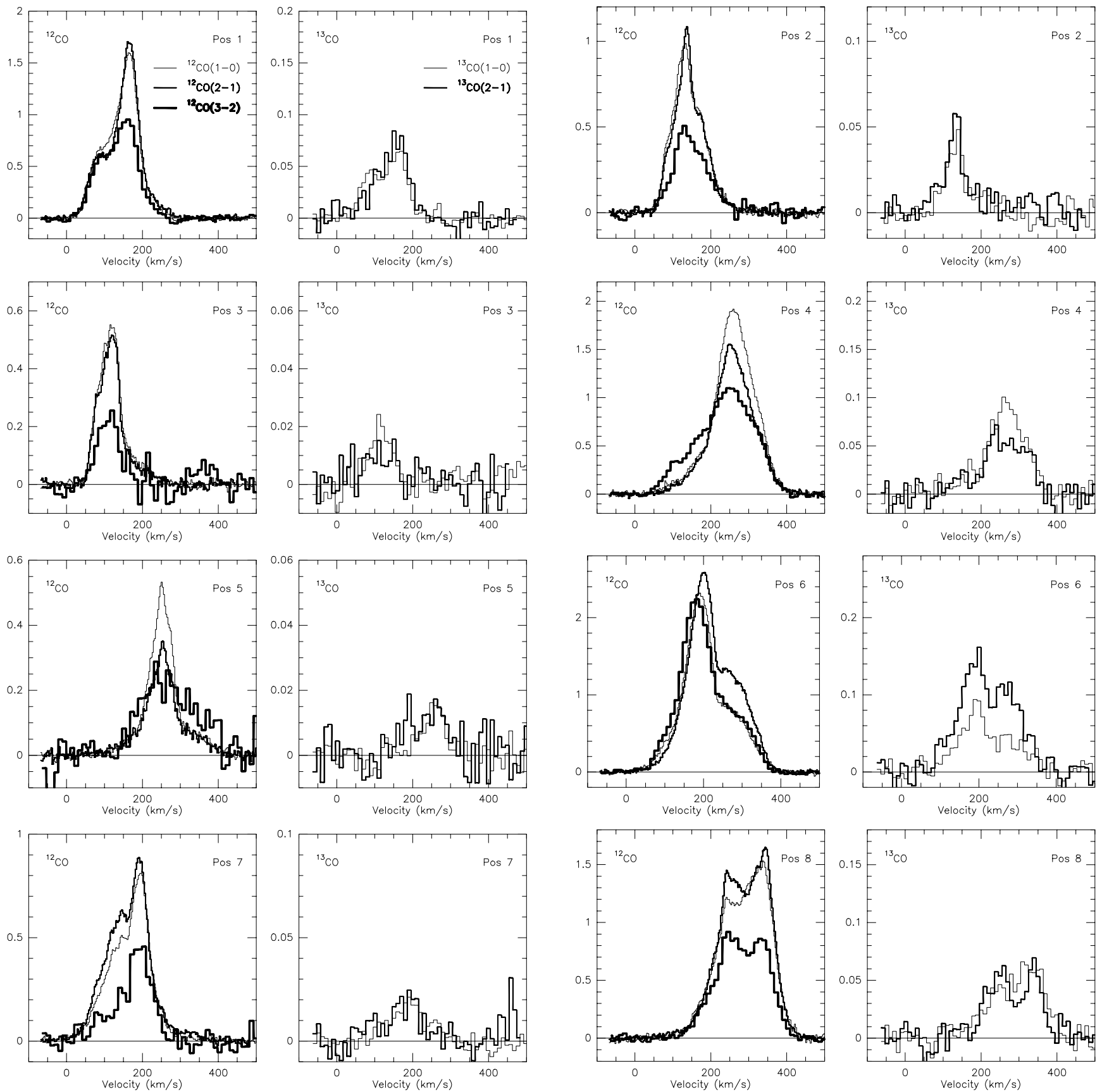

Fig. 2. Spectra of the $\mathrm{CO}(J=1 \rightarrow 0), \operatorname{CO}(J=2 \rightarrow 1)$, and $\mathrm{CO}(J=3 \rightarrow 2)$ (left), and the ${ }^{13} \mathrm{CO}(J=1 \rightarrow 0)$ and ${ }^{13} \mathrm{CO}(J=2 \rightarrow 1)($ right $)$ transitions at $22^{\prime \prime}$ resolution at individual positions. Line brightness temperatures are given in Kelvin on a $T_{\mathrm{mb}}$ scale. The $\operatorname{CO}(J=3 \rightarrow 2)$ spectra have been calculated from the data in Dumke et al. (2001).

transitions at individual positions are in good agreement except for the northern outflow (Pos. 4 and 5) where the wings of the $\mathrm{CO}(J=3 \rightarrow 2)$ line are broader than the corresponding profiles for the $\mathrm{CO}(J=1 \rightarrow 0)$ and $\mathrm{CO}(J=2 \rightarrow 1)$ lines (see Fig. 2). We attribute these line profile differences to small pointing offsets between the observations by Dumke et al. and our observations.

We have used our high-resolution CO map (see Fig. 1) and the beam patterns of the $30 \mathrm{~m}$ telescope (Greve et al. 1998) to estimate the contribution from the error-beam in each spectrum. Using the first two error-beam patterns we find that the contribution is negligible at all positions $(<5 \%)$.
The integrated and peak brightness temperature ratios agree well within the observational errors. In the following we use the integrated brightness temperature ratios, since this provides a higher signal to noise ratio for the ${ }^{13} \mathrm{CO}$ data. All $R$ ratios used throughout this paper are defined in Table 2. Only in the case of $R_{31}$ in the northern outflow (Pos. 4 and 5) we used the peak brightness temperature ratio, as the integrated $\operatorname{CO}(J=3 \rightarrow 2)$ intensities are too high due to the different line profile (see above). Statistical errors are negligible for all CO lines. From our $\mathrm{W} 3 \mathrm{OH}$ scans we estimate the systematic error of the $R_{10}$ ratio to be $\approx 15 \%$. The different observing methods at $3 \mathrm{~mm}$ 

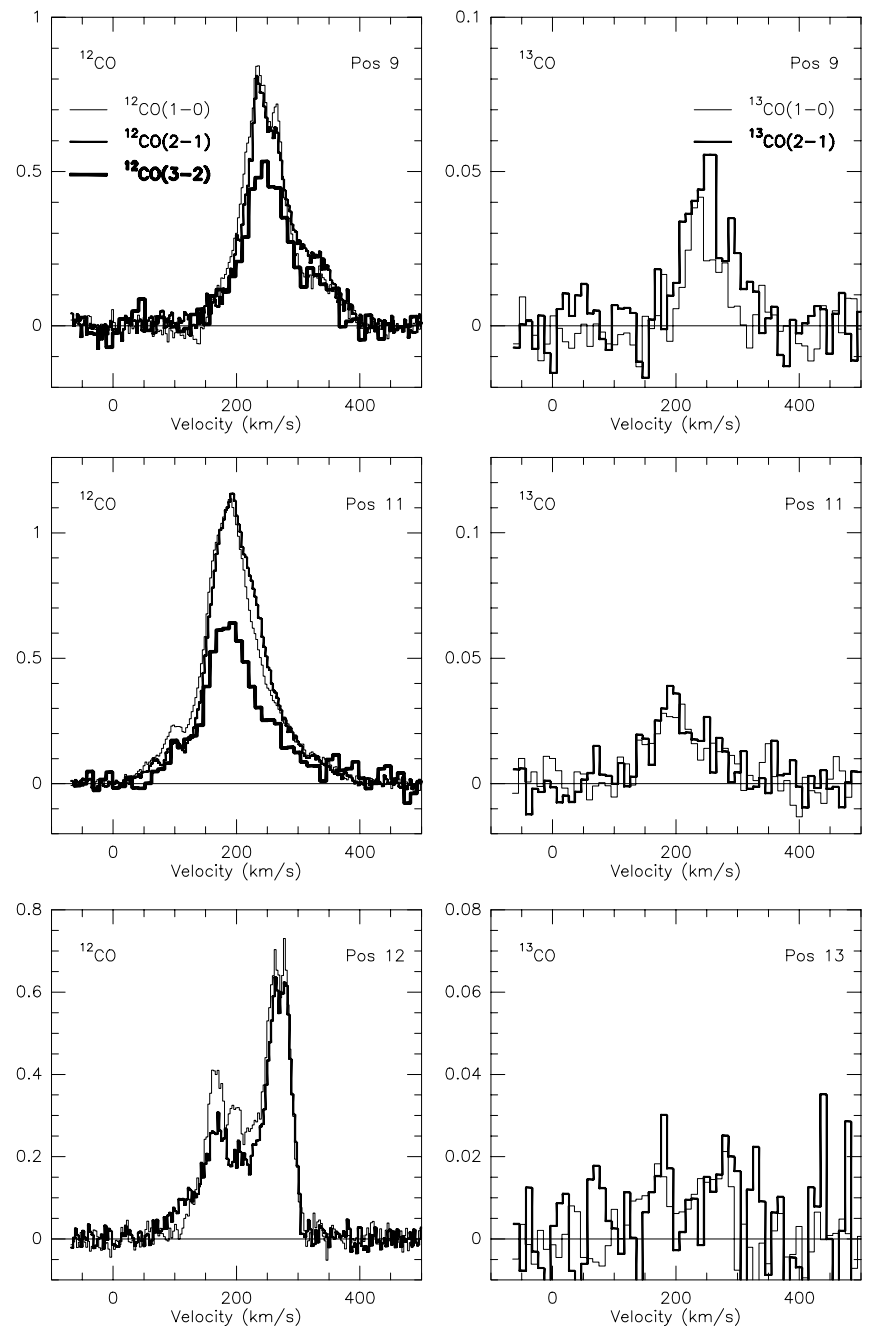
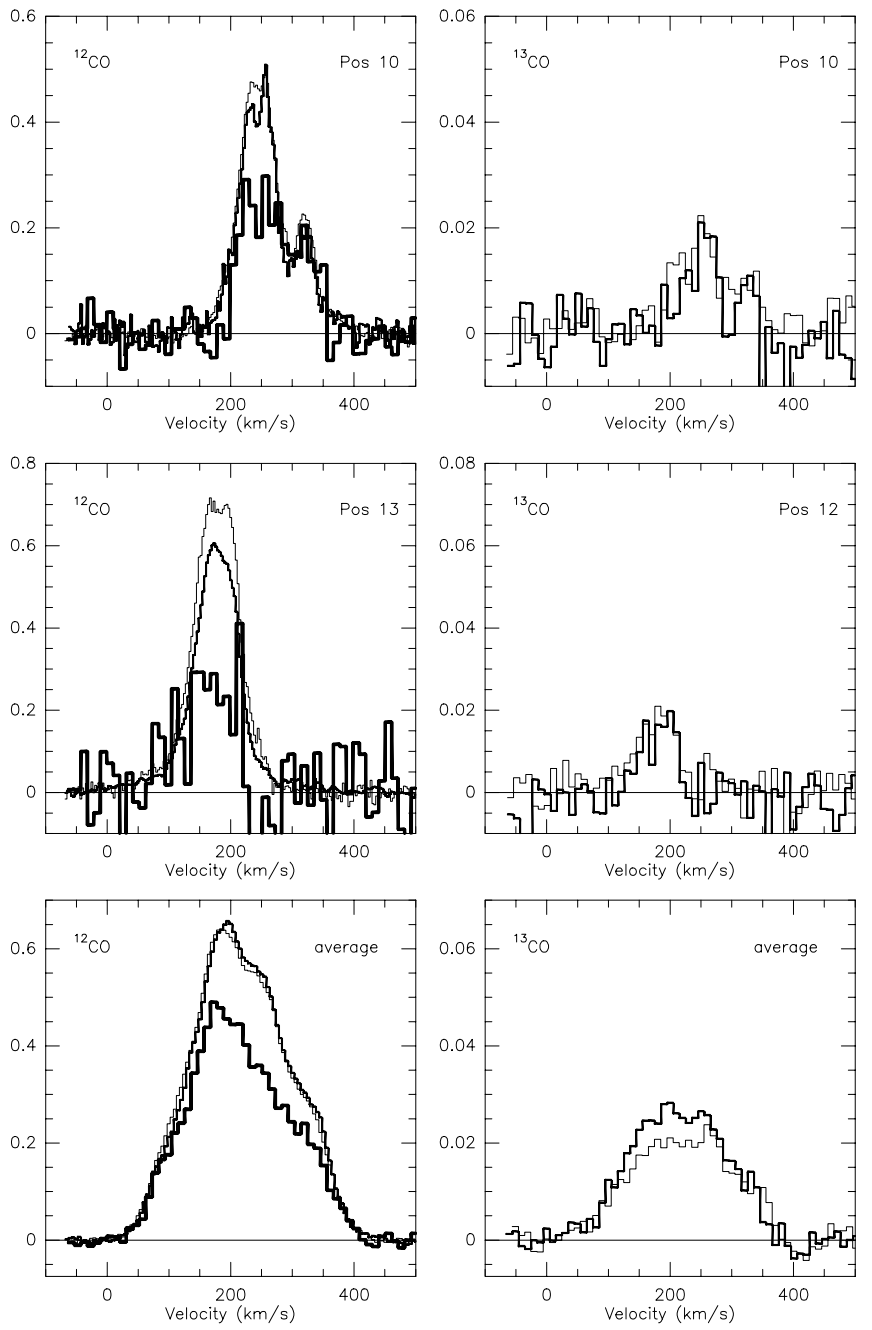

Fig. 2. continued. Note that the $\operatorname{CO}(J=3 \rightarrow 2)$ spectrum at position 11 has a offset of $-5^{\prime \prime}, 0^{\prime \prime}$ with respect to the other lines due the limited extend of the $\mathrm{CO}(J=3 \rightarrow 2)$ cube. The spectra at the bottom right panels are average spectra from all positions.

and $1 \mathrm{~mm}$ lead to $\approx 30 \%$ systematical errors for $R_{21}$ and $R_{21}^{13}$. Taking the independent uncertainties of our $\mathrm{CO}(J=1 \rightarrow 0)$ intensities and the $\mathrm{CO}(J=3 \rightarrow 2)$ errors reported by Dumke et al. (2001) lead to $\approx 30 \%$ error for the $R_{31}$ ratio. Line brightness temperature ratios and their errors, taking the statistical error for the ${ }^{13} \mathrm{CO}$ lines into account, are summarized in Table 2.

Figure 3 shows the spatial variations of the most relevant line brightness temperature ratios. Differences between individual positions are remarkably small. The only clear trend visible in the spatial distribution of the line ratios is a decreasing $R_{31}$ with galactocentric distance. This trend is visible along the streamer S1 and S3 as well as along the northern and southern outflow. Along S2, however, $R_{31}$ is constant. All other ratios do not show a clear trend with increasing distance from the central starburst region. $R_{21}$ is around unity for all positions covering the streamer system, slightly above unity in the southern, but only about 0.8 in the northern outflow. The corresponding ratios in ${ }^{13} \mathrm{CO}, R_{21}^{13}$, show a larger scatter and values range between 0.7 and 1.5. Judging from the ratio of the ground transitions of $\mathrm{CO}$ and ${ }^{13} \mathrm{CO}, R_{10}$, the analyzed positions can be subdivided into positions within and outside the optical disk. Within the disk, along the molecular streamers S1 and S2 and at the base of the northern and southern outflow the ratio $R_{10}$ is in the range of 20-26. Outside the optical disk, at the end the outflows and within the streamer system S3 and S4 the ratio rises to value between 29-39. While the line ratios at the base of the southern outflow almost resemble those in the molecular disk of M 82 (see Sect. 4.2), the gas in the northern outflow appears to be much less excited. In fact, $R_{21}$ and $R_{31}$ in the northern outflow are even lower than in the molecular streamers.

\subsection{Excitation conditions}

\subsubsection{Average properties}

To analyze the excitation conditions of the molecular gas surrounding M 82's starburst region we use a spherical, isothermal one component large velocity gradient (LVG) model. Given the small variations of the line brightness temperature ratios with position we use in the following the line ratios determined from the average spectra to investigate the global excitation away from the star forming regions. Note that these ratios differ from the numerical average of the line ratios at individual positions since they correspond to the intensity weighted averages which 
Table 1. CO line parameters at $22^{\prime \prime}$ resolution.

\begin{tabular}{rccccccccccc}
\hline \hline & $\begin{array}{c}T_{(1-0)}^{12} \\
{[\mathrm{mK}]}\end{array}$ & $\begin{array}{c}T_{(2-1)}^{12} \\
{[\mathrm{mK}]}\end{array}$ & $\begin{array}{c}{ }^{a} T_{(3-2)}^{12} \\
{[\mathrm{mK}]}\end{array}$ & $\begin{array}{c}T_{(1-0)}^{13}[\mathrm{mK}] \\
{\left[\begin{array}{cccccccc}T_{(2-1)}^{13} \\
{[\mathrm{mK}]}\end{array}\right.}\end{array}$ & $\begin{array}{c}W_{(1-0)}^{12} \\
{\left[\mathrm{~K} \mathrm{~km} \mathrm{~s}^{-1}\right]}\end{array}$ & $\begin{array}{c}W_{(2-1)}^{12} \\
{\left[\mathrm{~K} \mathrm{~km} \mathrm{~s}^{-1}\right]}\end{array}$ & $\begin{array}{c}{ }^{a} W_{(3-2)}^{12} \\
{\left[\mathrm{~K} \mathrm{~km} \mathrm{~s}^{-1}\right]}\end{array}$ & $\begin{array}{c}W_{(1-0)}^{13} \\
{\left[\mathrm{~K} \mathrm{~km} \mathrm{~s}^{-1}\right]}\end{array}$ & $\begin{array}{c}W_{(2-1)}^{13} \\
{\left[\mathrm{~K} \mathrm{~km} \mathrm{~s}^{-1}\right]}\end{array}$ & $\begin{array}{c}v_{\text {peak }} \\
{\left[\mathrm{km} \mathrm{s}^{-1}\right]}\end{array}$ \\
\hline 2 & 980 & 1690 & 930 & 60 & 70 & 155 & 153 & 107 & 6.7 & 7.0 & 165 \\
3 & 540 & 510 & 250 & 22 & 11 & 43 & 38 & 22 & 1.7 & 1.4 & 120 \\
4 & 1960 & 450 & 40 & 50 & 90 & 87 & 52 & 3.5 & 3.3 & 130 \\
5 & 520 & 340 & 200 & 15 & 10 & 44 & 33 & $b 35$ & 1.5 & 1.4 & 250 \\
6 & 2310 & 2580 & 2200 & 90 & 140 & 280 & 335 & 298 & 12 & 23 & 190 \\
7 & 810 & 880 & 440 & 20 & 19 & 90 & 104 & 45 & 2.3 & 2.0 & 195 \\
8 & 1510 & 1640 & 890 & 60 & 57 & 237 & 253 & 150 & 10 & 8.4 & 330 \\
9 & 840 & 780 & 500 & 38 & 52 & 74 & 74 & 54 & 3.1 & 4.3 & 235 \\
10 & 480 & 480 & 250 & 19 & 17 & 47 & 43 & 28 & 1.8 & 1.3 & 250 \\
11 & 1130 & 1150 & ${ }^{c} 620$ & 28 & 36 & 128 & 126 & ${ }^{2} 80$ & 3.6 & 3.5 & 190 \\
12 & 700 & 600 & 260 & 20 & 20 & 70 & 56 & 25 & 1.9 & 1.9 & 180 \\
13 & 690 & 610 & - & 17 & $<20$ & 65 & 56 & - & 2.2 & $<2.4$ & 270 \\
\hline Avg. & 655 & 645 & 480 & 20 & 26 & 98 & 100 & 74 & 3.6 & 4.8 \\
\hline
\end{tabular}

${ }^{a} \mathrm{CO}(J=3 \rightarrow 2)$ data from Dumke et al. (2001).

${ }^{b} \mathrm{CO}(J=3 \rightarrow 2)$ line profile wider than $\mathrm{CO}(J=1 \rightarrow 0)$ and $\mathrm{CO}(J=2 \rightarrow 1)$ line (see discussion in Sect. 3.1).

${ }^{c} \mathrm{CO}(J=3 \rightarrow 2)$ spectrum has a offset of $-5^{\prime \prime}, 0^{\prime \prime}$ from Pos. 11 .

would be observed if M 82 was shifted to cosmological distances and the emission would remain unresolved. Spatial variations of the excitation conditions are discussed in Sect. 3.2.2. The averaged spectra are shown at the bottom right panels of Fig. 2, the corresponding averaged integrated line ratios are given in the bottom row of Table 2 .

We compare LVG predicted line ratios to the observations in a LVG parameter space covering densities and kinetic temperatures of $\log \left(n_{\mathrm{H}_{2}}\right)=2.0-5.0 \mathrm{~cm}^{-3}$ and $T_{\text {kin }}=5-200 \mathrm{~K}$. $\mathrm{CO}$ abundances per velocity gradient and ${ }^{13} \mathrm{CO}$ abundances relative to $\mathrm{CO}$ are in the range of $[\mathrm{CO}] / \mathrm{d} v / \mathrm{d} r=1 \times 10^{-6}-2 \times$ $10^{-4} \mathrm{pc}\left(\mathrm{km} \mathrm{s}^{-1}\right)^{-1}$ and $[\mathrm{CO}] /\left[{ }^{13} \mathrm{CO}\right]=30-100$.

The observed CO ratios, $R_{21}$ and $R_{31}$, limit solutions in the LVG parameter space to a density range between $\log \left(n_{\mathrm{H}_{2}}\right)=$ $2.3-3.5 \mathrm{~cm}^{-3}$ where the lower density limit corresponds to the highest value of $[\mathrm{CO}] / \mathrm{d} v / \mathrm{d} r$ and the upper density limit to the lowest $[\mathrm{CO}] / \mathrm{d} v / \mathrm{d} r$. The measured high $R_{10}$ ratio restricts solutions to physical conditions with moderate opacities of the $\operatorname{CO}(J=1 \rightarrow 0)\left(\tau_{12} \approx 1-3\right)$ and very low opacities in the ${ }^{13} \mathrm{CO}(J=1 \rightarrow 0)$ transition $\left(\tau_{13} \approx 0.01-0.05\right)$ within this density range. As a consequence solutions are limited to small $\mathrm{CO}$ abundances per velocity gradient $(\leq 2 \times$ $\left.10^{-5} \mathrm{pc}\left(\mathrm{km} \mathrm{s}^{-1}\right)^{-1}\right)$ and $[\mathrm{CO}] /\left[{ }^{13} \mathrm{CO}\right]$ abundance ratios $>50$. This additional constraint on $[\mathrm{CO}] / \mathrm{d} v / \mathrm{d} r$ yields an allowed density range of $\log \left(n_{\mathrm{H}_{2}}\right)=2.7-3.5 \mathrm{~cm}^{-3}$ and small $\mathrm{CO}$ column densities per velocity interval $\left(<2 \times 10^{17} \mathrm{~cm}^{-2}\left(\mathrm{~km} \mathrm{~s}^{-1}\right)^{-1}\right)$. The kinetic temperature is not well constrained without assumptions on the values of the $[\mathrm{CO}] /\left[{ }^{13} \mathrm{CO}\right]$ abundance ratio and the $\mathrm{CO}$ abundance per velocity gradient. A lower limit on the kinetic temperature of $T_{\text {kin }} \geq 30 \mathrm{~K}$ is given by the densest possible solution within the parameter space.
In Figs. 4 and 5 we show LVG solutions for the averaged line ratios as a function of abundance and velocity gradient variations. The lines represent the temperature-density combinations for which the LVG predicted line ratios match the average observed line ratios. The figures show that lower $\mathrm{CO}$ abundances per velocity gradient and/or higher fractional ${ }^{13} \mathrm{CO}$ abundances lead to cooler, denser solutions while higher abundances per velocity gradients and/or lower fractional ${ }^{13} \mathrm{CO}$ abundances lead to warmer and more diffuse solutions, respectively. For a $\mathrm{CO}$ abundance per velocity gradient of $1 \times 10^{-5} \mathrm{pc}\left(\mathrm{km} \mathrm{s}^{-1}\right)^{-1}$ and a $[\mathrm{CO}] /\left[{ }^{13} \mathrm{CO}\right]$ abundance ratio of 80 the averaged line intensity ratios are well reproduced by a kinetic temperate of $T_{\text {kin }}=55 \mathrm{~K}$ with a $\mathrm{H}_{2}$ density of $\log \left(n_{\mathrm{H}_{2}}\right)=3.2 \mathrm{~cm}^{-3}$. These values correspond to the intersection of the lines shown in Figs. 4 and 5 (center).

\subsubsection{Individual regions}

In the following we compare the excitation conditions at individual positions. To do so, we assume that the abundance and velocity gradient variations do not change with position and fix the $\mathrm{CO}$ abundances per velocity gradient and the fractional ${ }^{13} \mathrm{CO}$ abundance to $1 \times 10^{-5} \mathrm{pc}\left(\mathrm{km} \mathrm{s}^{-1}\right)^{-1}$ and 80 respectively.

Along the streamers S1 and S2 the excitation conditions are fairly similar. The decrease of the $R_{31}$ along $\mathrm{S} 1$ is reflected in a small decrease of the density and temperature from $\log \left(n_{\mathrm{H}_{2}}\right)=3.2 \mathrm{~cm}^{-3}$ and $T_{\text {kin }}=50 \mathrm{~K}$ at the base of the streamer to $\log \left(n_{\mathrm{H}_{2}}\right)=3.1 \mathrm{~cm}^{-3}$ and $T_{\text {kin }}=40 \mathrm{~K}$ at its end. Similar conditions are met along streamer S2 $\left(\log \left(n_{\mathrm{H}_{2}}\right) \approx 3.3-3.1 \mathrm{~cm}^{-3}\right.$ and $T_{\text {kin }} \approx 45-40 \mathrm{~K}$ ). The base of the northern outflow (Pos. 4) can be fit well with cooler gas $\left(T_{\text {kin }} \approx 30 \mathrm{~K}\right)$ at comparable 
Table 2. Selected integrated line brightness temperature ratios at $22^{\prime \prime}$ resolution. Errors include $15 \%$ systematic uncertainty for $R_{10}, 30 \%$ for $R_{21}$ and $R_{21}^{13}$ and $30 \%$ for $R_{32}$. Statistical errors have been included for the ${ }^{13} \mathrm{CO}$ lines only.

\begin{tabular}{rccccccccc}
\hline \hline & \multirow{2}{*}{$\alpha$} & $\delta$ & $\frac{W(\mathrm{CO}(2-1))}{W(\mathrm{CO}(1-0))}$ & $\frac{W(\mathrm{CO}(3-2))}{W(\mathrm{CO}(1-0))}$ & $\frac{W\left({ }^{13} \mathrm{CO}(2-1)\right)}{W\left({ }^{13} \mathrm{CO}(1-0)\right)}$ & $\frac{W(\mathrm{CO}(1-0))}{W\left({ }^{13} \mathrm{CO}(1-0)\right)}$ & $\frac{W(\mathrm{CO}(2-1))}{W\left({ }^{13} \mathrm{CO}(2-1)\right)}$ & $\frac{W(\mathrm{CO}(2-1))}{W\left({ }^{13} \mathrm{CO}(1-0)\right)}$ & Region \\
& \multicolumn{2}{c}{$\mathrm{J} 2000.0$} & & $R_{21}$ & $R_{31}$ & $R_{21}^{13}$ & $R_{10}$ & $R_{2}^{12 / 13}$ & $R_{21}^{12 / 13}$ \\
\hline 1 & $09: 55: 43.9$ & $69: 40: 41$ & $1.0 \pm 0.3$ & $0.7 \pm 0.2$ & $1.0 \pm 0.4$ & $23 \pm 5$ & $22 \pm 5$ & $23 \pm 5$ & $\mathrm{~S} 1$ \\
2 & $09: 55: 40.1$ & $69: 40: 30$ & $1.0 \pm 0.3$ & $0.6 \pm 0.1$ & $0.9 \pm 0.4$ & $26 \pm 6$ & $26 \pm 4$ & $25 \pm 5$ & $\mathrm{~S} 1$ \\
3 & $09: 55: 38.4$ & $69: 40: 11$ & $0.9 \pm 0.3$ & $0.5 \pm 0.1$ & $0.8 \pm 0.2$ & $25 \pm 6$ & $27 \pm 6$ & $22 \pm 5$ & $\mathrm{~S} 1$ \\
4 & $09: 55: 51.3$ & $69: 41: 06$ & $0.8 \pm 0.25$ & ${ }^{a} 0.6 \pm 0.2$ & $0.7 \pm 0.2$ & $20 \pm 4$ & $24 \pm 5$ & $16 \pm 5$ & $\mathrm{O}-\mathrm{N}$ \\
5 & $09: 55: 50.3$ & $69: 41: 27$ & $0.7 \pm 0.15$ & ${ }^{a} 0.4 \pm 0.15$ & $0.9 \pm 0.4$ & $29 \pm 7$ & $24 \pm 12$ & $22 \pm 5$ & $\mathrm{O}-\mathrm{N}$ \\
6 & $09: 55: 53.5$ & $69: 40: 30$ & $1.2 \pm 0.3$ & $1.1 \pm 0.2$ & $1.5 \pm 0.6$ & $22 \pm 6$ & $19 \pm 4$ & $28 \pm 6$ & $\mathrm{O}-\mathrm{S}$ \\
7 & $09: 55: 55.9$ & $69: 40: 12$ & $1.1 \pm 0.3$ & $0.5 \pm 0.2$ & $0.9 \pm 0.4$ & $39 \pm 10$ & $54 \pm 10$ & $45 \pm 9$ & $\mathrm{O}-\mathrm{S}$ \\
8 & $09: 55: 58.4$ & $69: 40: 55$ & $1.0 \pm 0.3$ & $0.6 \pm 0.2$ & $0.8 \pm 0.4$ & $24 \pm 6$ & $30 \pm 5$ & $25 \pm 5$ & $\mathrm{~S} 2$ \\
9 & $09: 56: 01.3$ & $69: 41: 02$ & $1.0 \pm 0.3$ & $0.7 \pm 0.2$ & $1.4 \pm 0.5$ & $24 \pm 6$ & $17 \pm 3$ & $24 \pm 5$ & $\mathrm{~S} 2$ \\
10 & $09: 56: 04.6$ & $69: 41: 17$ & $0.9 \pm 0.3$ & $0.6 \pm 0.2$ & $0.7 \pm 0.4$ & $26 \pm 9$ & $33 \pm 5$ & $24 \pm 5$ & $\mathrm{~S} 2$ \\
11 & $09: 56: 01.1$ & $69: 40: 35$ & $1.0 \pm 0.3$ & ${ }^{b} 0.6 \pm 0.15$ & $1.0 \pm 0.5$ & $36 \pm 10$ & $36 \pm 8$ & $35 \pm 8$ & $\mathrm{~S} 3$ \\
12 & $09: 56: 03.7$ & $69: 40: 17$ & $0.8 \pm 0.25$ & $0.4 \pm 0.1$ & $1.0 \pm 0.4$ & $36 \pm 14$ & $29 \pm 6$ & $29 \pm 6$ & $\mathrm{~S} 3$ \\
13 & $09: 56: 05.2$ & $69: 40: 43$ & $0.9 \pm 0.25$ & - & $<1.1$ & $30 \pm 10$ & $>23$ & $25 \pm 5$ & $\mathrm{~S} 4$ \\
\hline
\end{tabular}

${ }^{a}$ Peak brightness temperature ratio (see text).

${ }^{b} \mathrm{CO}(J=3 \rightarrow 2)$ spectrum has a offset of $-5^{\prime \prime}, 0^{\prime \prime}$ from Pos. 11 .

${ }^{c}$ Intensity weighted average determined from the integrated line brightness temperature ratios of the averaged spectra (see Fig. 2 bottom right panels).

density $\left(\log \left(n_{\mathrm{H}_{2}}\right)=3.1 \mathrm{~cm}^{-3}\right)$ while the high $R_{10}$ ratio combined with the low $\mathrm{CO}$ ratios, $R_{21}$ and $R_{31}$, at the end of the outflow leads to $T_{\text {kin }} \approx 40 \mathrm{~K}$ with a low density $\log \left(n_{\mathrm{H}_{2}}\right)=$ $2.8 \mathrm{~cm}^{-3}$. In contrast, the base of the southern outflow consists of denser gas at higher temperature $\left(T_{\text {kin }} \approx 70 \mathrm{~K}, \log \left(n_{\mathrm{H}_{2}}\right) \approx\right.$ $3.5 \mathrm{~cm}^{-3}$ ), comparable to conditions in the starburst disk (see Sect. 4.2). At the end of the southern outflow as well as along the streamers S3 and S4 (the positions with the highest $R_{10}$ ratio) a warm diffuse medium is required to match the observed line ratios. The temperatures and densities range from $T_{\text {kin }} \approx$ $60-120 \mathrm{~K}$ and $\log \left(n_{\mathrm{H}_{2}}\right)=2.7-3.0 \mathrm{~cm}^{-3}$ respectively. Note that the large difference in $R_{21}$ and $R_{31}$ at the end of the southern outflow (Pos. 7; 1.1 and 0.5 respectively) can not be fit by a single gas component. In Fig. 6 we show where the different regions lie in the LVG temperature density space. Not all line ratios at a given positions can be fit equally well and the locations of the regions show roughly the best agreement with the measured line ratios.

\section{Discussion}

\subsection{Properties of the streamer/outflow gas}

The high isotope line ratio observed in the $J=1-0$ transition $\left(R_{10}\right)$ at all positions clearly rules out that the gas conditions in the outer parts of M 82 are similar to those in the disk of the Milky Way or other spiral galaxies. Typically the $\mathrm{CO}(J=1 \rightarrow 0)$ line has large optical depth in disk clouds in spiral galaxies and even the emission in ${ }^{13} \mathrm{CO}(J=1 \rightarrow 0)$ is only moderately optically thin which results in $R_{10}$ typically about 5. $R_{10}$ much closer to the $[\mathrm{CO}] /\left[{ }^{13} \mathrm{CO}\right]$ abundance ratio is a well studied signature of molecular gas in the centers of active galaxies (including M 82's starburst disk with $R_{10} \approx 20$ ) and mergers (e.g. Aalto et al. 1991; Sage \& Isbell 1991; Henkel $\&$ Mauersberger 1993). The reason for the ${ }^{13} \mathrm{CO}$ depression in luminous starburst galaxies is still under debate but most explanations involve ${ }^{13} \mathrm{CO} / \mathrm{CO}$ abundance ratio variations and opacity effects (see e.g. Henkel \& Mauersberger 1993; Aalto et al. 1995; Kikumoto et al. 1998; Tanigucchi et al. 1999; Paglione et al. 2001; Meier \& Turner 2004). Given its origin from the star forming disk and the likely interaction with outflowing ionized gas, high $R_{10}$ ratios are therefore expected from the gas located in northern and southern molecular outflow. The observed increase of $R_{10}$ along both outflows supports the idea that "superwinds" have large isotope line ratios (Tanigucchi et al. 1999). For gas along the streamers $\mathrm{S} 1$ and $\mathrm{S} 2$ the high $R_{10}$ ratios are unexpected as $R_{10}$ is usually found to decrease with galactocentric distance (Paglione et al. 2001) and since both streamers are located well outside the central star forming regions. We note, however, that decreases of $R_{10}$ typically only becomes significant for galactocentric distances larger than $2 \mathrm{kpc}$ (Paglione et al. 2001). Increasing $R_{10}$ ratios in the vicinity of starburst have also been observed in e.g. IC 342 (Wright et al. 1993) and NGC 6946 (Meier \& Turner 2004). In these cases the large isotopic line ratio has been attribute to dispersed low-density gas in the "spray regions" of barred potentials. Given the low density in S1 and S2 and that these streamers form the base of the disrupted H I features within M 82's disk (Yun et al. 1993) we speculate that their large $R_{10}$ may result from dispersed gas due to tidal forces along S1 and S2. 


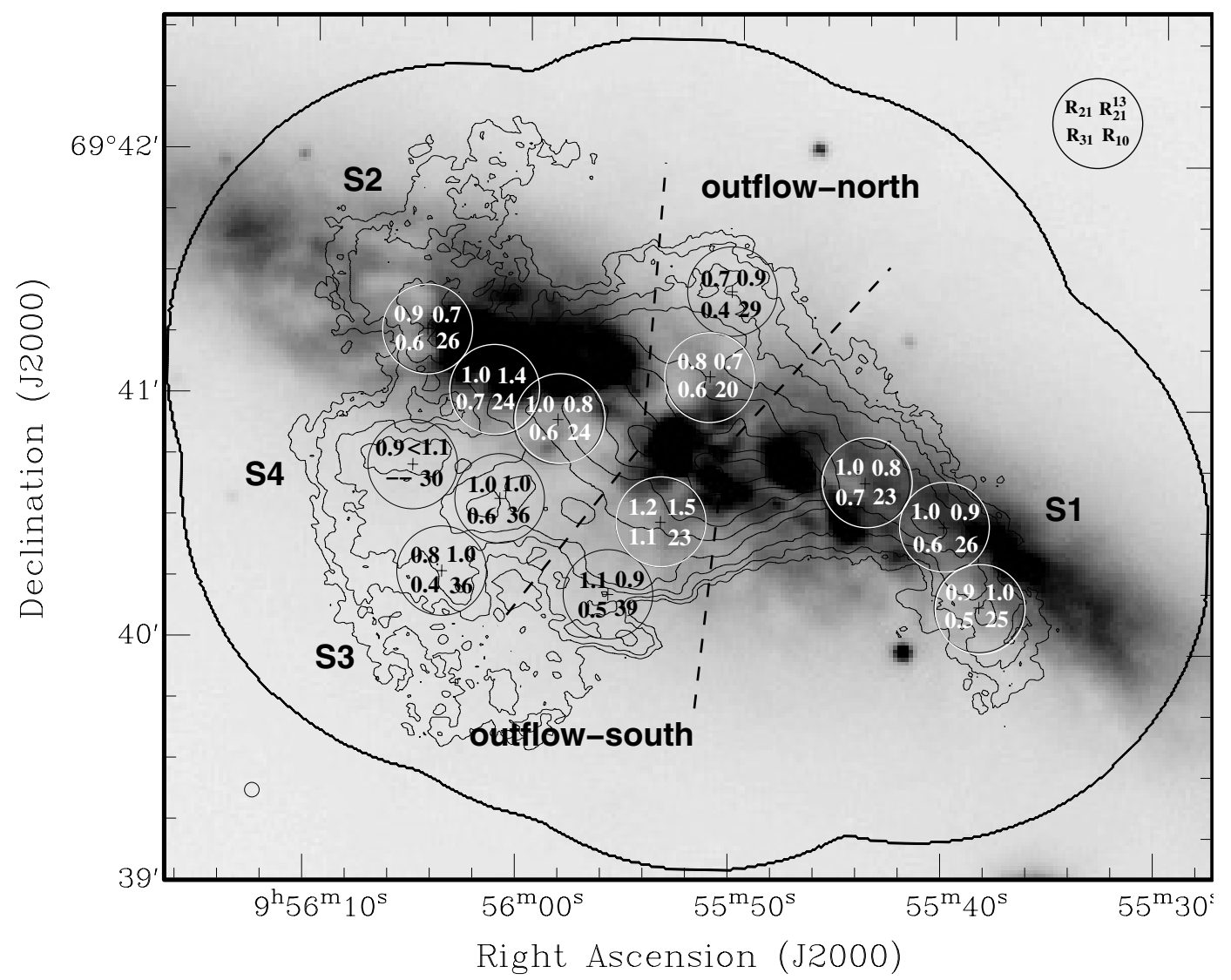

Fig. 3. Spatial variations of the $R_{21}$ (upper left number), $R_{31}$ (lower left), $R_{21}^{12 / 13}$ (upper right) and $R_{10}$ (lower right) line brightness temperature ratios (see Table 2 for definitions). The greyscale is an optical $V$-band image of M 82 (Cheng et al. 1997). The contours represent the distribution of the $\mathrm{CO}(J=1 \rightarrow 0)$ emission (see Fig. 1).
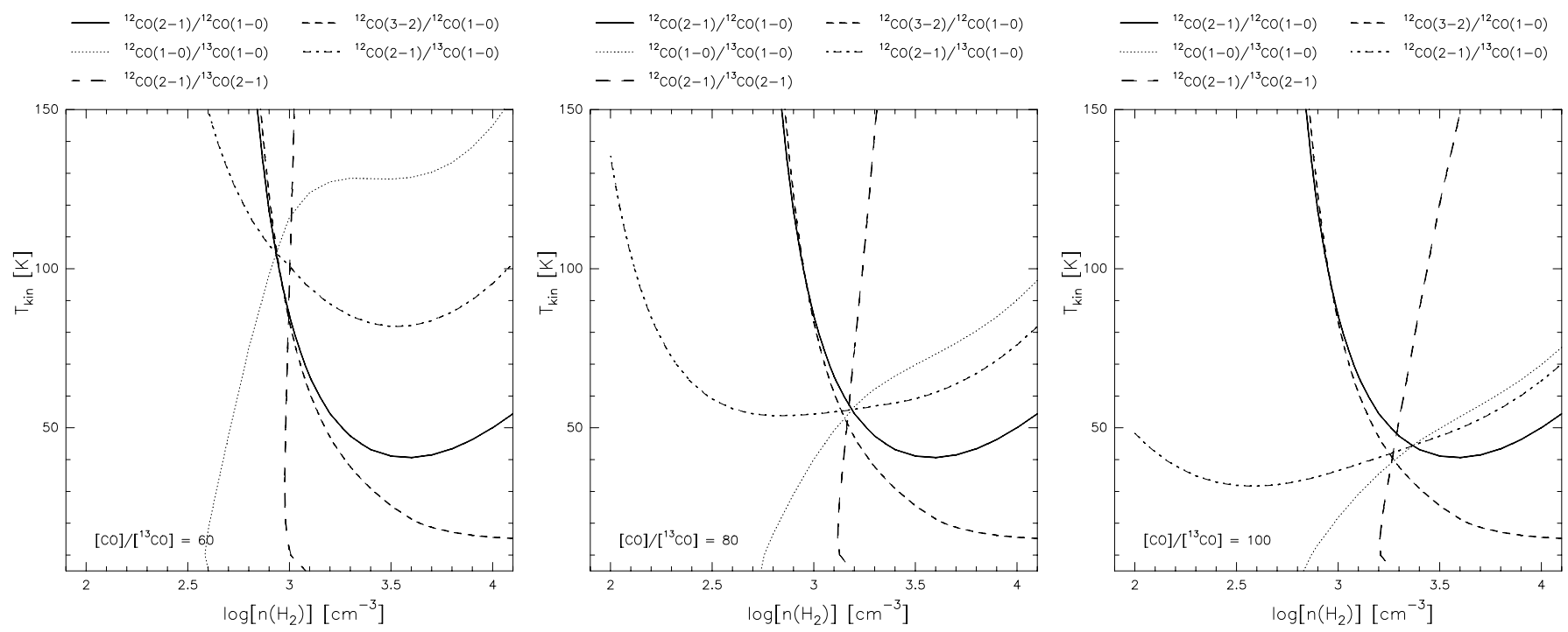

Fig. 4. LVG line brightness temperature ratios as a function of the kinetic temperature and $\mathrm{H}_{2}$ density for a fractional ${ }^{13} \mathrm{CO}$ abundance of 60 , 80 and 100. The $\mathrm{CO}$ abundance per velocity gradient is $1 \times 10^{-5} \mathrm{pc}\left(\mathrm{km} \mathrm{s}^{-1}\right)^{-1}$ for each graph. Displayed line ratios correspond to the averaged observed values listed in the bottom row of Table 2.

In the context of our LVG models, the large isotopic line ratio is mainly a result of the very low optical depth of the ground transition of ${ }^{13} \mathrm{CO}\left(\tau_{13} \approx 0.01-0.05\right)$. These low opacities result from the low ${ }^{13} \mathrm{CO}$ abundances per velocity gradient of our models. Outside the optical disk, in regions with $R_{10}>30$, higher kinetic temperatures and lower $\mathrm{H}_{2}$ densities (resulting in lower column densities per velocity interval) push ${ }^{13} \mathrm{CO}$ opacities to the lower end of the range. For gas in local thermodynamic equilibrium (LTE) and large optical depth in $\operatorname{CO}(J=$ $1 \rightarrow 0$ ) the ratio is given by $R_{10} \approx \tau_{13}^{-1}$. Although (i) the 


\section{$-{ }^{12} \mathrm{CO}(2-1) / /^{12} \mathrm{CO}(1-0) \quad---{ }^{12} \mathrm{CO}(3-2) /^{12} \mathrm{CO}(1-0)$ $\quad \ldots \ldots \ldots . . .{ }^{12} \mathrm{CO}(1-0) / /^{13} \mathrm{CO}(1-0) \quad \ldots \quad{ }^{12} \mathrm{CO}(2-1) /{ }^{13} \mathrm{CO}(1-0)$ $-{ }^{12} \mathrm{CO}(2-1) / /^{13} \mathrm{CO}(2-1)$}

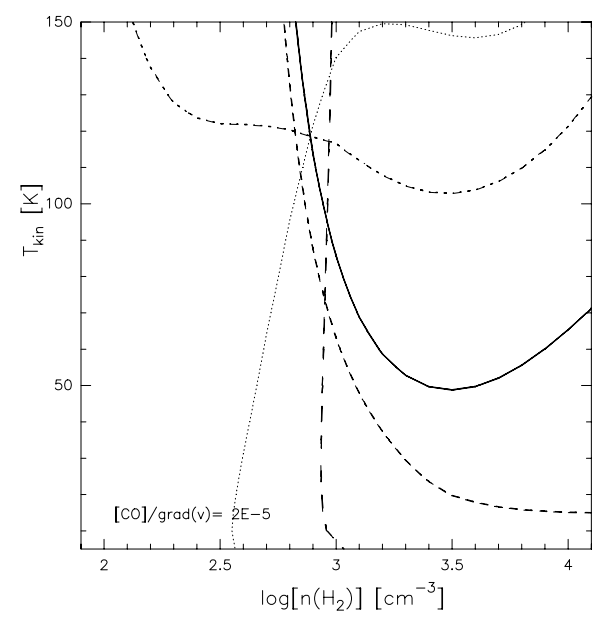

$\begin{array}{llll}{ }^{12} \mathrm{CO}(2-1) /{ }^{12} \mathrm{CO}(1-0) & \ldots & { }^{12} \mathrm{CO}(3-2) / /^{12} \mathrm{CO}(1-0) \\ \ldots \ldots \ldots . . . & { }^{12} \mathrm{CO}(1-0) /{ }^{13} \mathrm{CO}(1-0) & \ldots . & { }^{12} \mathrm{CO}(2-1) / /^{13} \mathrm{CO}(1-0)\end{array}$ $-{ }^{12} \mathrm{CO}(2-1) /{ }^{13} \mathrm{CO}(2-1)$

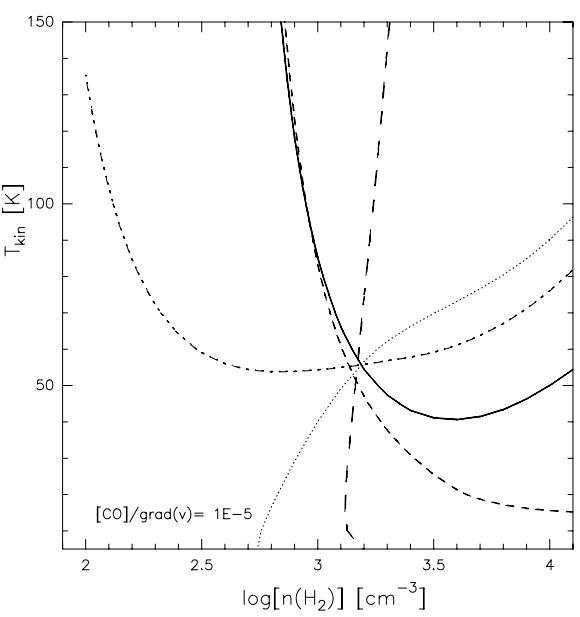

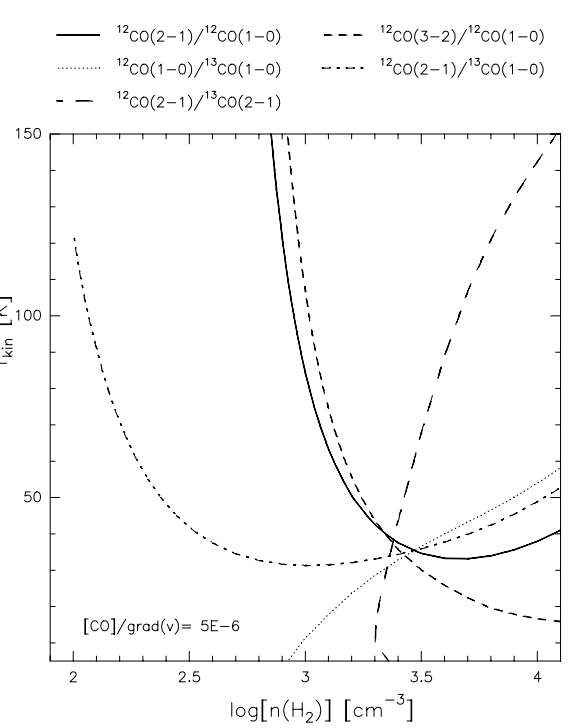

Fig. 5. LVG line brightness temperature ratios as a function of the kinetic temperature and $\mathrm{H}_{2}$ density for a $\mathrm{CO}$ abundance per velocity gradient of $2 \times 10^{-5}, 1 \times 10^{-5}$ and $5 \times 10^{-6} \mathrm{pc}\left(\mathrm{km} \mathrm{s}^{-1}\right)^{-1}$. The fractional ${ }^{13} \mathrm{CO}$ abundance is 80 for each graph. Displayed line ratios correspond to the averaged observed values listed in the bottom row of Table 2 .

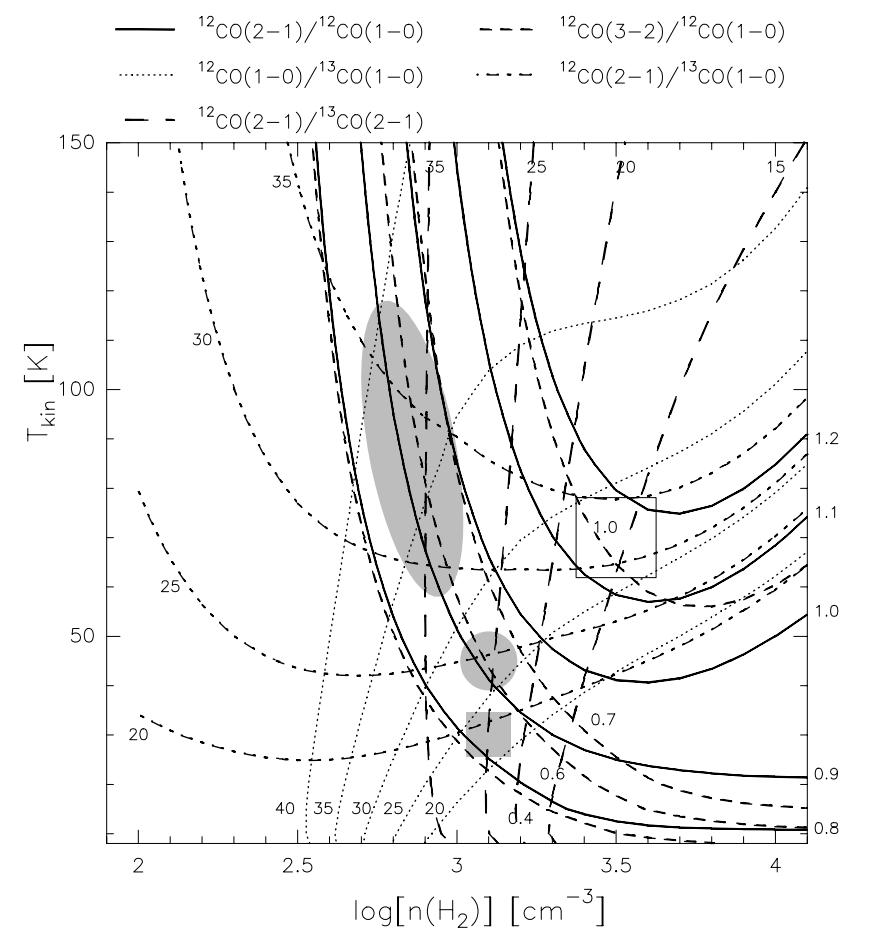

Fig. 6. LVG predicted $R_{21}, R_{31}, R_{10}, R_{21}^{12 / 13}$ and $R_{2}^{12 / 13}$ line ratios as a function of $\mathrm{H}_{2}$ density and kinetic temperature for a $\mathrm{CO}$ abundance per velocity gradient of $1 \times 10^{-5} \mathrm{pc}\left(\mathrm{km} \mathrm{s}^{-1}\right)^{-1}$ and a fractional ${ }^{13} \mathrm{CO}$ abundance of 80 . Numbers indicate the respective line ratios. The symbols show the density - temperature combination of the streamers S1 and S2 (grey circle), the northern outflow (grey square), the base of the southern outflow (white square) and regions south of the optical disk (S3, S4, and the end of the southern outflow; grey ellipse).

excitation temperatures between $\mathrm{CO}$ and ${ }^{13} \mathrm{CO}$ differ up to a factor of 2 in our models (non LTE) and (ii) the moderate opacities in $\operatorname{CO}(J=1 \rightarrow 0)$ introduce an additional deviation from the above equation, the opacity in ${ }^{13} \mathrm{CO}$ is the most important quantity for variations in $R_{10}$. This suggests that the CO emission outside the optical disk (streamers S3 and S4 and at the end of both outflows) arises from diffuse and presumably warm gas with low opacities in ${ }^{13} \mathrm{CO}$.

Interestingly, the southern and northern outflows have different excitation conditions (mainly reflected in the low $R_{21}$ and $R_{31}$ ratios in the northern outflow). The lower temperatures towards the north might indicate that the gas is less affected by outflowing ionized gas. Indeed, the outflow seen in $\mathrm{H} \alpha$ and $\mathrm{X}$-rays is not symmetrical with respect to the optical disk but brighter and also more extended towards the south (e.g. Lehnert et al. 1999). This, however, might simply be an effect of the orientation of the outflow with respect to the observer since the southern outflow is inclined towards us (see e.g. McKeith et al. 1995; Greve 1998, for the outflow geometry).

Although the kinetic temperatures of the molecular gas are difficult to constrain without better estimates of the $\mathrm{CO}$ abundances, the general picture to emerge from the LVG models is that most of the gas in the outer regions of M 82 has low densities and low optical depth at temperatures in excess of $30 \mathrm{~K}$.

\subsection{Comparison to M 82's center}

The physical conditions of the gas within the central molecular toroid have been the subject of many studies (e.g. Wild et al. 1992; Güsten et al. 1993; Mao et al. 2000; Petitpas \& Wilson 2000; Weiß et al. 2001; Ward et al. 2003). These studies have shown, that the observed line ratios can be reproduced by emission from a low (LE) and a high excitation (HE) gas component (Güsten et al.1993; Mao et al. 2000; Weiß et al. 2001; Ward et al. 2003). In this picture the high excitation component, responsible for the excitation of levels beyond the $J=$ $4 \rightarrow 3$ transition, arise from dense gas at temperatures similar to or higher than the dust temperature $\left(\log \left(n_{\mathrm{H}_{2}}\right) \approx 3.5-4.5 \mathrm{~cm}^{-3}\right.$; $T_{\text {kin }} \geq 40 \mathrm{~K}$ ). The low excitation component is emitted by diffuse gas at much lower density $\left(\log \left(n_{\mathrm{H}_{2}}\right) \approx 3.0 \mathrm{~cm}^{-3}\right)$. 
Table 3. Observed and model predicted CO integrated flux densities in the center and outer parts of M 82 .

\begin{tabular}{cccrrrrr}
\hline \hline Transition & $\begin{array}{c}\text { Center } \\
{\left[\mathrm{Jy} \mathrm{km} \mathrm{s}^{-1}\right]}\end{array}$ & $\begin{array}{c}\text { Outer } \\
{\left[\mathrm{Jy} \mathrm{km} \mathrm{s}^{-1}\right]}\end{array}$ & $\begin{array}{r}\text { Model center } \\
{\left[\mathrm{Jy} \mathrm{km} \mathrm{s}^{-1}\right]}\end{array}$ & $\begin{array}{r}\text { Model outer } \\
{\left[\mathrm{Jy} \mathrm{km} \mathrm{s}^{-1}\right]}\end{array}$ & $\begin{array}{c}\text { Model total } \\
{\left[\mathrm{Jy} \mathrm{km} \mathrm{s}^{-1}\right]}\end{array}$ & $\begin{array}{c}I_{\text {center }} / I_{\text {total }} \\
{[\%]}\end{array}$ & Ref. \\
\hline $\mathrm{CO}(1-0)$ & $5.1 \mathrm{E} 3 \pm 5.0 \mathrm{E} 2$ & $1.5 \mathrm{E} 4 \pm 2.8 \mathrm{E} 3$ & 6320 & 17100 & 23420 & 27 & 1 \\
$\mathrm{CO}(2-1)$ & $2.2 \mathrm{E} 4 \pm 5.0 \mathrm{E} 3$ & $7.0 \mathrm{E} 4 \pm 1.8 \mathrm{E} 4$ & 24300 & 65710 & 90010 & 27 & 1 \\
$\mathrm{CO}(3-2)$ & $4.9 \mathrm{E} 4 \pm 8.0 \mathrm{E} 3$ & $9.7 \mathrm{E} 4 \pm 2.0 \mathrm{E} 4$ & 46090 & 103040 & 149130 & 31 & 2 \\
$\mathrm{CO}(4-3)$ & $6.4 \mathrm{E} 4 \pm 1.3 \mathrm{E} 4$ & - & 64210 & 90570 & 154780 & 41 & 3 \\
$\mathrm{CO}(5-4)$ & - & - & 78110 & 45310 & 123420 & 63 & \\
$\mathrm{CO}(6-5)$ & ${ }^{a} 9.7 \mathrm{E} 4 \pm 2.4 \mathrm{E} 4$ & - & 87360 & 13650 & 101010 & 86 & 4 \\
$\mathrm{CO}(7-6)$ & ${ }^{a} 7.4 \mathrm{E} 4 \pm 2.3 \mathrm{E} 4$ & - & 78920 & 2960 & 81880 & 96 & 3 \\
$\mathrm{CO}(8-7)$ & - & - & 45570 & 470 & 46040 & 99 & \\
$\mathrm{CO}(9-8)$ & - & - & 13210 & 43 & 13250 & 100 & \\
\hline
\end{tabular}

Ref. 1: Weiß et al. (2001) (30 m data); 2: Dumke et al. (2001); 3: Mao et al. (2000); 4: Ward et al. (2003).

${ }^{a}$ Total flux covered by the observations.

Our analysis shows that the gas surrounding M 82's starburst region has similar properties as the low excitation component in the starburst center itself. Similar conclusions have been reached from an analysis of the $\mathrm{CO} J=3 \rightarrow 2 / J=2 \rightarrow 1$, ${ }^{13} \mathrm{CO} / \mathrm{CO} J=3 \rightarrow 2$ and $\mathrm{C}^{18} \mathrm{O} / \mathrm{CO}$ ratios in the molecular "halo" by Seaquist \& Clark (2001). They interpret their decreasing line ratios as decreasing $\mathrm{CO}$ excitation and optical depth with increasing distance from the nuclear region. These findings are in line with our results. Seaquist \& Clark find that the variations of the observed line ratios in the transition region between the center and the "halo" are consistent with a decreasing filling factor of the high excitation component with distance from the center. In this picture the $\mathrm{CO}$ emission from the outer regions arise exclusively from gas with similar properties than the low excitation component in the center. We discuss this scenario in more detail in the next section. This suggests that mainly the diffuse low density molecular gas in the starburst region is involved in the outflow. Given the higher inertia of dense (and therefore more massive) gas clouds, we speculate that the superwind affects the kinematics of gas at low density more strongly. However, the high $\mathrm{CO}$ excitation found at the base of the southern outflow indicates that also gas at higher density might be pushed out of the starburst regions, at least to scale heights of a few hundred pc, less than half the distance seen in the outflow of the diffuse molecular gas.

\subsection{CO-line-SED}

\subsubsection{High $J$ CO fluxes}

We have used published maps in various $\mathrm{CO}$ transitions towards M 82 to estimate the total flux density emitted in each line from the center and the outer parts of the $\mathrm{CO}$ distribution. Large-scale maps (covering the streamer/outflow system) only exist up to the $\operatorname{CO}(J=3 \rightarrow 2)$ transition. The observed flux densities from the outer parts of M 82 were determined by masking the central molecular disk in the $\operatorname{CO}(J=1 \rightarrow 0)$ and $\mathrm{CO}(J=2 \rightarrow 1)$ IRAM $30 \mathrm{~m}$ data cubes from Weiß et al. (2001) and the $\operatorname{CO}(J=3 \rightarrow 2)$ data cube from Dumke et al. (2001) (after smoothing all data cubes to $22^{\prime \prime}$ spatial resolution and regridding onto the same grid). The mask was chosen to contain a flux density of $\approx 5000 \mathrm{Jy} \mathrm{km} \mathrm{s}^{-1}$ in the $\mathrm{CO}(J=1 \rightarrow 0)$ data cube (see Fig. 1), close to the value determined by us for the central molecular disk (Walter et al. 2002). CO flux densities from the center were determined within the inverted mask. For the center we also analyzed the $\mathrm{CO}(J=4 \rightarrow 3)$ and $\operatorname{CO}(J=7 \rightarrow 6)$ cubes published by Mao et al. (2000). Since the $\mathrm{CO}(J=4 \rightarrow 3)$ map is larger than our mask we applied the same technique as for the lower $J$ lines to determine the central flux density in $\mathrm{CO}(J=4 \rightarrow 3)$. The $\mathrm{CO}(J=7 \rightarrow 6)$ map is smaller than the central mask and we adopt here the total flux density in the data cube to be representative for the flux density from the center. The central flux density of the $\operatorname{CO}(J=6 \rightarrow 5)$ transition was taken from the map by Ward et al. (2003), which roughly covers the central mask. The observed CO flux densities for the center and the outer parts are summarized in Table 3.

\subsubsection{CO line SED of the starburst disk}

In Fig. 7 (left) we show the observed CO line SED (CO integrated flux density vs. the rotational quantum number) in the center of M 82. CO flux densities rise up to the $J=6 \rightarrow 5$ transition, although we can not rule out that the true $\operatorname{CO}(J=$ $7 \rightarrow 6$ ) flux density is higher due to the limited coverage of the $J=7 \rightarrow 6$ map. In this plot we also show the LVG predicted CO line SED for a high (HE) and a low excitation (LE) component in the center. Following the approach by Seaquist \& Clark we used the LVG model derived from the average line ratios in the steamer/outflow system as LE component (LE: $\log \left(n_{\mathrm{H}_{2}}\right)=3.2 \mathrm{~cm}^{-3}, T_{\text {kin }}=55 \mathrm{~K}, \mathrm{CO}$ abundance per velocity gradient $1 \times 10^{-5} \mathrm{pc}\left(\mathrm{km} \mathrm{s}^{-1}\right)^{-1}$; see Sect.3.2.1). Adding a HE component with $\log \left(n_{\mathrm{H}_{2}}\right)=4.2 \mathrm{~cm}^{-3}, T_{\text {kin }}=50 \mathrm{~K}$, similar to conditions in the molecular lobes (e.g. Weiß et al. 2001), results in a good fit to the observed CO line SED of the center. For these models we adopted a filling factor of 1/20 and $1 / 15$ for the HE and the LE component in a solid angel 

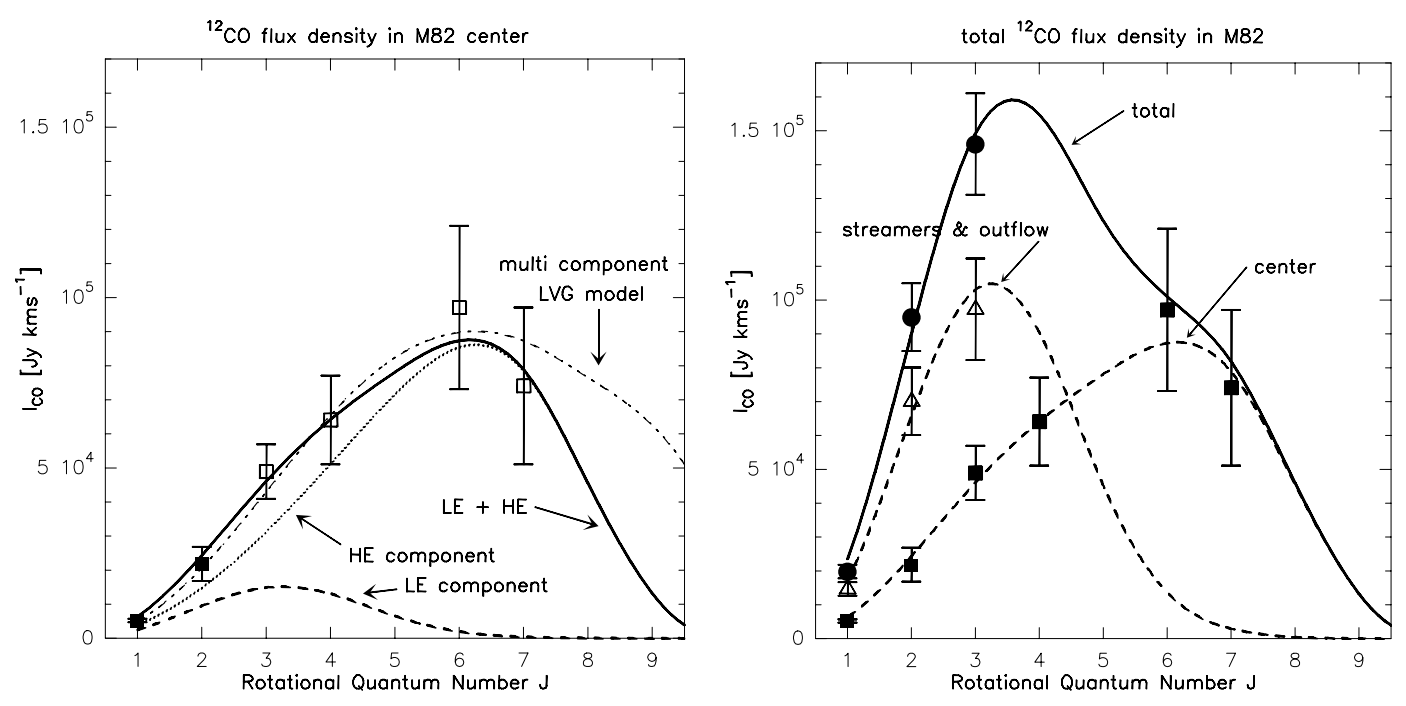

Fig. 7. Left: observed and model predicted CO line SED for the center of M 82 using a two component LVG model. The dotted line shows the predicted CO line SEDs of a high (HE), the lower dashed line of a low (LE) excitation component. The LE model correspond to the physical conditions determined in the outer regions of the CO distribution, the HE component is similar to the conditions in the molecular lobes. The black line is the sum of both components. For comparison we also show the CO line SED for a multi component LVG model (dashed line, Weiß et al. 2001). The filled squares are those CO observations which have been used to constrain the multi component model. Open squares are CO flux densities compiled from the literature. Right: CO line SED for M 82 including the molecular streamers and outflow. The dashed lines show the model for the center (same as solid line to the left) and for the streamers and outflow. The solid line shows the total predicted CO line $\mathrm{SED}$ in the central $3 \times 3 \mathrm{kpc}$. Filled rectangles are observed flux densities from the center of M 82, open circles the observed flux densities in the streamer/outflow system and filled circles the total observed flux density in the central $3 \times 3 \mathrm{kpc}$ of M 82 . Observed and model predicted line flux densities are given in Table 3.

of $\Omega_{\text {center }} \approx 1000 \operatorname{arcsec}^{2}$ (as defined by our mask for the center). For comparison we show the CO line SED in the center of M 82 predicted by a high resolution LVG analysis based on the $J=1-0$ and $J=2-1$ transitions only (Weiß et al. 2001). The fluxes have been calculated taking all individual LVG solutions within the center into account. This example shows, that LVG models based on the low- $J$ lines of $\mathrm{CO}$ and their rare isotopes give surprisingly good estimates of the flux density in the high- $J$ CO lines.

\subsubsection{CO line SED of total emission}

In a similar way we have calculated the CO line SED for the streamer/outflow system. Here we used the LE component with an area filling factor of $1 / 30$ (derived from the average $\mathrm{CO}(J=1 \rightarrow 0)$ spectrum at $22^{\prime \prime}$ resolution and the LVG predicted line brightness temperature) and a source solid angle of $\Omega_{\text {outer }} \approx 14000 \operatorname{arcsec}^{2}$ (estimated from the zero-spacing corrected $\mathrm{CO}(J=1 \rightarrow 0)$ OVRO map). The predicted line flux densities are shown together with the observed values in Fig. 7 (right). The plot demonstrates that this simple two phase model for the center and the outer regions is in good agreement with the observations. More detailed studies of the gas in the center show that the LE component is most likely warmer than the $55 \mathrm{~K}$ used here (e.g. Weiß et al. 2001; Ward et al. 2003). Higher temperatures at sightly lower density, however, do not change the predicted flux densities in the LE component significantly. In any case the line SED in the center is dominated by the dense gas that excites the high- $J$ CO lines. This is in line with CO models for the center of NGC 253, where the CO line
SED is fit by a single high excitation gas component (Bradford et al. 2003; Bayet et al. 2004).

The combined $C O$ line $S E D$ from the center and the streamer/outflow system, which would be observed if M 82 was shifted to cosmological distances, however differs strongly from that derived from the center alone (see Fig. 7 right). The large area of the surrounding gas makes it the dominant component in the emission of the low- $J$ CO transitions. Since this gas is not dense enough to produce significant flux beyond the $\mathrm{CO}(J=5 \rightarrow 4)$ line, its $\mathrm{CO}$ line SED peaks already at the $\mathrm{CO}(J=3 \rightarrow 2)$ transition. Consequently, even though twothirds of the $\mathrm{CO}(J=1 \rightarrow 0)$ flux density arises from regions outside the star forming disk, $\mathrm{CO}$ lines above $\mathrm{CO}(J=5 \rightarrow 4)$ transition are mainly emitted from the center. In the combined CO line SED, flux densities rise up to the $J=4 \rightarrow 3$ transition and decrease beyond this line. We summarize the $\mathrm{CO}$ flux densities derived from the LVG models in Table 3. Table 3 also gives the fraction of the total flux density emitted by the center in each $\mathrm{CO}$ transition. For comparison with other studies we give the model predicted line fluxes in Table 4.

\subsection{CO line SEDs at high redshift}

Currently CO line SEDs in sources at redshift $>2$ are still poorly constrained. A few sources, however, have been observed in more than two CO transitions which allow at least for a quantitative comparison with the CO line SED of M 82. Among the best studied examples are the Cloverleaf quasar $(z=2.5$, Barvainis et al. 1997; Weiß et al. 2003), IRAS FSC $10214(z=2.3$, Brown \& Vanden Bout 1992; 
Table 4. LVG model predicted CO fluxes in the center $(\Omega=2.35 \times$ $\left.10^{-8} \mathrm{sr}\right)$ and outer $\left(\Omega=3.29 \times 10^{-7} \mathrm{sr}\right)$ parts of M 82 .

\begin{tabular}{cccc}
\hline \hline Transition & Center & Outer & Total \\
& \multicolumn{3}{c}{$10^{-16} \mathrm{~W} \mathrm{~m}^{-2}$} \\
\hline $\mathrm{CO}(1-0)$ & 0.2 & 0.7 & 0.9 \\
$\mathrm{CO}(2-1)$ & 1.9 & 5.1 & 6.9 \\
$\mathrm{CO}(3-2)$ & 5.3 & 11.9 & 17.2 \\
$\mathrm{CO}(4-3)$ & 9.8 & 13.9 & 23.8 \\
$\mathrm{CO}(5-4)$ & 15.0 & 8.7 & 23.7 \\
$\mathrm{CO}(6-5)$ & 20.2 & 3.1 & 23.3 \\
$\mathrm{CO}(7-6)$ & 21.2 & 0.8 & 22.0 \\
$\mathrm{CO}(8-7)$ & 14.0 & 0.1 & 14.2 \\
$\mathrm{CO}(9-8)$ & 4.6 & 0.01 & 4.6 \\
\hline
\end{tabular}

Solomon et al. 1992), PSS 2322+1944 $(z=4.1$, Cox et al. 2002; Carilli et al.2002a), BR1202-0725 ( $z=4.7$ Omont et al. 1996; Carilli et al. 2002b) and J1148+5251 $(z=6.4$, Walter et al. 2003; Bertoldi et al. 2003). In all these sources the CO flux densities are rising with increasing rotational quantum numbers (at least up to the $J=6 \rightarrow 5$ transitions), similar to the center of M 82 and NGC 253. These studies argue against large extended low excitation $\mathrm{CO}$ halos surrounding these objects at high redshifts. However, there are a few examples where the flux density from the $\mathrm{CO} J=3 \rightarrow 2$ transition is similar or higher than that of the $J=7 \rightarrow 6$ line (see e.g. SMM $14011+0252(z=2.5$, Downes \& Solomon 2003), SMM 16368+4057 $(z=2.4$, Neri et al. 2003). In light of our new results in M 82 we suggest that the low $J=7 \rightarrow 6 / J=$ $3 \rightarrow 2$ ratios in these sources may not necessarily be caused by a different excitation of the central molecular gas concentration, but may result from an additional more extended and diffuse gas reservoir.

\section{Summary}

We have presented observations of the $J=1 \rightarrow 0$ and $J=$ $2 \rightarrow 1$ transition of $\mathrm{CO}$ and ${ }^{13} \mathrm{CO}$ towards 13 selected regions outside the central starburst disk of M 82 covering the prominent molecular streamers and the $\mathrm{CO}$ outflow. Our observations show that the $J=2 \rightarrow 1 / J=1 \rightarrow 0$ and $J=3 \rightarrow 2 / J=1 \rightarrow 0$ ratios of $\mathrm{CO}$ are lower in all streamer/outflow regions than in the central molecular disk. The $\mathrm{CO}$ ratios are fairly constant along the analyzed positions and show only a slight trend of decreasing ratios with increasing distance from the center. The isotope line ratio $\mathrm{CO}(J=1 \rightarrow 0) /{ }^{13} \mathrm{CO}(J=1 \rightarrow 0)$ is around 25 along the most prominent molecular streamers, similar to values found in the starburst disk, and increases along the outflows to 35. Similarly high values for this ratio are found in the diffuse streamers S3 and S4 south-east of the optical disk of M 82.

An LVG analysis of the gas suggests that the low CO ratios combined with the high isotope line ratio are best explained by a low density $\left(\log \left(n_{\mathrm{H}_{2}}\right) \approx 3.0 \mathrm{~cm}^{-3}\right)$ molecular environment with kinetic temperatures in excess of $30 \mathrm{~K}$ and $\left[{ }^{13} \mathrm{CO}\right] /[\mathrm{CO}]$ abundance ratios $\sim 80$. In this picture the high $\mathrm{CO}(J=1 \rightarrow 0) /{ }^{13} \mathrm{CO}(J=1 \rightarrow 0)$ ratio is mainly caused by very low opacities in the ${ }^{13} \mathrm{CO}$ lines. Our analysis does not allow to rule out denser gas at lower kinetic temperature if abundance variations are taken into account. However densities in excess of $\log \left(n_{\mathrm{H}_{2}}\right) \approx 3.5 \mathrm{~cm}^{-3}$ require $[\mathrm{CO}] /\left[{ }^{13} \mathrm{CO}\right]$ abundance ratios $>100$ or very small $\mathrm{CO}$ abundances per velocity gradient $\left([\mathrm{CO}] / \mathrm{d} v / \mathrm{d} r<1 \times 10^{-6}\right)$ to explain the observed line ratios which we consider unlikely.

We use the LVG predicted line brightness temperature ratios of the $\mathrm{CO} J>3$ transitions to estimate the total flux density emitted in the high- $J$ lines from the diffuse $C O$ component surrounding M 82 starburst region. We find that the density of the streamer/outflow gas is not high enough to produce significant flux above the $J=5 \rightarrow 4$ line. As a consequence the $\mathrm{CO}$-line SED of the outer gas drops off beyond $J=3 \rightarrow 2$ line while it is rising at least up to the $J=6 \rightarrow 5$ line in the central molecular disk. The total $J=1 \rightarrow 0$ and $J=2 \rightarrow 1$ flux density within the central $3 \times 3 \mathrm{kpc}$, however, is clearly dominated by the emission from the outer regions. This implies that one has to exercise caution when comparing global, unresolved, high- $z$ line ratios to the values measured in the centers only of nearby starburst galaxies. We conclude that small high- $J /$ low- $J$ CO ratios ratios seen in some high- $z$ sources are not necessarily caused by a different excitation of the central molecular gas concentration, but may result from an additional more extended and diffuse gas reservoir around these systems.

Acknowledgements. We thank the referee, Dr. M.S. Yun, for valuable suggestions which helped to improve this paper. We also thank Drs. M. Dumke and R. Q. Mao for making available their data to us. This paper is based on observations carried out at the IRAM $30 \mathrm{~m}$ telescope. IRAM is supported by INSU/CNRS (France), MPG (Germany) and IGN (Spain).

\section{References}

Aalto, S., Black, J. H., Johansson, L. E. B., \& Booth, R. S. 1991, A\&A, 249,323

Aalto, S., Booth, R. S., Black, J. H., \& Johansson, L. E. B. 1995, A\&A, 300, 369

Barvainis, R., Maloney, P., Antonucci, R., \& Alloin, D. 1997, ApJ, 484, 695

Bayet, E., Gerin, M., Phillips, T. G., \& Contursi, A. 2004, A\&A, 427, 45

Bertoldi, F., Cox, P., Neri, R., et al. 2003, A\&A, 409, L47

Bradford, C. M., Nikola, T., Stacey, C. J., et al. 2003, ApJ, 586, 891

Brown, R. L., \& Vanden Bout, P. A. 1992, ApJ, 397, L19

Carilli, C. L., Kohno, K., Kawabe, R., et al. 2002a, AJ, 123, 1838

Carilli, C. L., Cox, P., Bertoldi, F., et al. 2002b, ApJ, 575, 145

Cheng, K.-P., Collins, N., Angione, R., et al. 1997, UV/Visible Sky Gallery

Cox, P., Omont, A., Djorgovski, S. G., et al. 2002, A\&A, 387, 406

Downes, D., \& Solomon, P. M. 2003, ApJ, 528, 37

Dumke, M., Nieten, C., Thuma, G., Wielebinski, R., \& Walsh, W. 2001, A\&A, 373, 853

Güsten, R., Serabyn, E., Kasemann, C., et al. 1993, ApJ, 402, 537

Greve, A., Kramer, C., \& Wild, W. 1998, A\&A, 133, 271

Greve, A. 2004, A\&A, 416, 67

Henkel, C., \& Mauersberger, R. 1993, A\&A, 274, 730

Johansson, L. E. B., Olofsson, H., Hjalmarson, A., Gredel, R., \& Black, J. H. 1994, A\&A, 291, 89 
Kikumoto, T., Taniguchi, Y., Nakai, N., et al. 1998, PASJ, 50, 309

Lehnert, M. D., Heckman, T. M., \& Weaver, K. A. 1999, ApJ, 523, 575

Mao, R. Q., Henkel, C., Schulz, A., et al. 2000, A\&A, 358, 433

McKeith, C. D., Greve, A., Downes, D., \& Prada, F. 1995, A\&A, 293, 703

Meier, D. S., \& Turner, J. L. 2004, AJ, 127, 2069

Neri, R., Genzel, R., Ivison, R. J., et al. 2003, ApJ, 597, L113

Omont, A., McMahon, R. G., Cox, P., et al. 1996, A\&A, 315, 1

Paglione, T. A. D, Wall, W. F., Young, J. S., et al. 2001, ApJS, 135, 183

Diamond, P., Wills, K. A., Wilkinson, P. N., \& Alef, W. 1999, MNRAS, 307, 761

Petitpas, G. R., \& Wilson, C. D. 2000, ApJ, 538, L117

Sage, L. J., \& Isbell, D. W. 1991, A\&A, 247, 320

Seaquist, E. R., \& Clark, J. 2001, ApJ, 552, 133

Solomon, P. M., Downes, D., \& Radford, S. J. E. 1992, ApJ, 398, L29
Stark, A. A., \& Carlson, E. R. 1984, ApJ, 279, 122

Tanigucchi, Y., Ohyama, Y., \& Sanders, D. B. 1999, ApJ, 522, 214

Taylor, C. L., Walter, F., \& Yun, M. S. 2001, ApJ, 562, L43

Walter, F., Bertoldi, F., Carilli, C. L., et al. 2003, Nature, 424, 406

Walter, F., Weiß, A., \& Scoville, N. Z. 2002, ApJ, 580, L21

Ward, J. S., Zmuidzinas, J., Harris, A., \& Isaak, K. G. 2003, ApJ, 587, 171

Weiß, A., Walter, F., Neininger, N., \& Klein, U. 1999, A\&A, 345, L23 Weiß, A., Neininger, N., Hüttemeister, S., \& Klein, U. 2001, A\&A, 365,571

Weiß, A., Henkel, C., Downes, D., \& Walter, F. 2003, A\&A, 409, L41

Wild, W., Harris, A. I., Eckart, A., et al. 1992, A\&A, 265, 447

Wright, M. C. H., Ishizuki, S., Turner, J. L., Ho, P. T. P., \& Lo, K. Y. 1993, ApJ, 406, 470

Young, J. S., \& Scoville, N. Z. 1984, ApJ, 287, 153

Yun, M. S., Ho, P. T. P., \& Lo, K. Y. 1993, ApJ, 411, 17

Yun, M. S., Carilli, C. L., Kawabe, R., et al. 2000, ApJ, 528, 171 